\title{
Once-Weekly Administration of Dapsone/Pyrimethamine vs. Aerosolized Pentamidine as Combined Prophylaxis for Pneumocystis carinii Pneumonia and Toxoplasmic Encephalitis in Human Immunodeficiency Virus-Infected Patients
}

\author{
Milos Opravil, Bernard Hirschel, Adriano Lazzarin, \\ Alison Heald, Marc Pechère, Sigmund Rüttimann, \\ Anne Iten, Jan von Overbeck, Daniel Oertle, \\ Gerard Praz, Dominique A. Vuitton, \\ Franco Mainini, and Ruedi Lüthy
}

\author{
From the Universit. Hospitals of Zurich, Geneva. Basel, Lausanne, and \\ Bern. Switzerland: Kantonsspital St. Gallen and Institut Central des \\ Hopitaux Valaisans. Sion. Switzerland; H. San Raffaele and Clinica \\ Malattie Infettive Ospedale L. Sacco. Milan. Italy; and Universit!' \\ Hospital Besançon. France
}

\begin{abstract}
To evaluate combined prophylaxis for Pneumocystis carinii pneumonia (PCP) and toxoplasmic encephalitis, 533 patients with symptomatic human immunodeficiency virus infection and/ or CD4 lymphocyte counts of $<200 / \mu \mathrm{L}$ were randomized to receive dapsone/pyrimethamine (200/75 mg once weekly) or aerosolized pentamidine (300 mg every 4 weeks). The median CD4 lymphocyte count was $110 / \mu \mathrm{L} ; 47.5 \%$ were seropositive for toxoplasma antibodies. The median duration of follow-up was 483 days. In the intent-to-treat analysis, 12 cases of PCP and 14 of toxoplasmic encephalitis occurred in the dapsone/pyrimethamine group and 13 and 20 cases, respectively, in the aerosolized pentamidine group (adjusted relative risk for toxoplasmosis, $0.56 ; P=.10$ ). However, only two of the 14 cases of toxoplasmic encephalitis in the dapsone/ pyrimethamine group developed during actual treatment. The mortality among the two groups was similar. Dapsone/pyrimethamine was not tolerated by $30 \%$ of participants. A subanalysis of 240 matched, tolerant patients yielded a relative risk for toxoplasmosis of $0.21(P=.014)$, a result favoring the use of dapsone/pyrimethamine. Dapsone/pyrimethamine was as effective as aerosolized pentamidine as prophylaxis for PCP and significantly reduced the incidence of toxoplasmic encephalitis among those participants who tolerated it.
\end{abstract}

Prophylaxis for opportunistic infections has had a strong impact on the management of immunosuppressed human immunodeficiency virus (HIV)-infected patients in recent years, and epidemiological data indicate that Pneumocystis carinii pneumonia (PCP) has become less frequent as a consequence of prevention [1]. Aerosolized pentamidine became available in Switzerland in 1987 and was subsequently proven effective for both primary and secondary prophylaxis for PCP in controlled trials [2, 3]. Among systemic prophylactic agents, trimethoprim-sulfamethoxazole became widely used [4] and was later shown to be more effective but also more toxic than aerosolized pentamidine $[5,6]$. Other combi-

Received 18 March 1994; revised 27 June 1994.

Grant support: Swiss National AIDS Research Program (grant numbers 90-7051 and 91-7059).

Presented in part at the 9th International Conference on AIDS/IV STD World Congress, held in Berlin. Germany, in 1993 [abstract number POB10-1429].

Written informed consent was obtained from all patients and the study was approved by the ethics committees of all participating centers.

Current addresses: Dr. Heald, Division of Infectious Diseases, Duke University Medical Center, Durham, NC 27710; Dr. Rüttimann, Medizinische Abteilung, Kantonsspital, 8208 Schafthausen, Switzerland; and Dr. Oertle, Medizinische Abteilung. Spital Limmattal, 8952 Schlieren, Switzerland.

Reprints or correspondence: Dr. M. Opravil, Division of Infectious Diseases, University Hospital, 8091 Zurich, Switzerland.

Clinical Infectious Diseases 1995;20:531-41

(C) 1995 by The University of Chicago. All rights reserved

1058-4838/95/2003-0008\$02.00 nations, such as dapsone and trimethoprim [7] or pyrimethamine and sulfadiazine [8], have been used for treatment of PCP before, but sparse data exist concerning their prophylactic efficacy [9]. Only recently, the prophylactic efficacy of the combination of dapsone and pyrimethamine against PCP and toxoplasmosis was demonstrated [10].

Since in $>95 \%$ of all AIDS patients with toxoplasmic encephalitis there is serological evidence of previous infection with Toxoplasma gondii [10-13], reactivation of a latent infection is the principal pathogenetic step in the development of toxoplasmic encephalitis during immunodeficiency. Considering the increasing frequency of toxoplasmic encephalitis among AIDS-defining opportunistic infections [1] and the $50 \%$ seroprevalence of toxoplasma antibodies in Switzerland [14], primary prophylaxis for toxoplasmic encephalitis seems desirable and clinically important, at least for seropositive patients.

The sequential inhibition of dihydrofolate reductase by pyrimethamine and of dihydropteroate synthetase by sulfonamides and dapsone is the rationale for a combination regimen that blocks the folate metabolism in $P$. carinii and $T$. gondii. Dapsone and pyrimethamine have been active against both organisms in vitro and in murine models [1517]; therefore, this combination was chosen for evaluation as combined prophylaxis for PCP and toxoplasmic encephalitis. Low cost and the potential for once-weekly administration [18] are additional attractive features of the dapsone/ pyrimethamine combination. As aerosolized pentamidine 
was the standard prophylactic agent against PCP at the time the trial was designed, we planned a prospective, randomized comparison of dapsone/pyrimethamine and aerosolized pentamidine administered as primary and secondary prophylaxis for PCP and as primary prophylaxis for toxoplasmic encephalitis.

\section{Methods}

\section{Study Population}

The participants were adults with HIV infection and an immunodeficiency score of at least 5 . This score, for selecting patients with a high risk for PCP $[2,19]$, was determined according to the following clinical and laboratory criteria: 5 points were assigned for a peripheral blood CD4 lymphocyte count of $<200 / \mu \mathrm{L}$ or AIDS (except Kaposi's sarcoma); 3 points for symptomatic HIV infection without AIDS; and 2 points for Kaposi's sarcoma, a CD4 lymphocyte count of $200-500 / \mu \mathrm{L}$, or positivity for HIV p24 antigen in serum. Previous PCP, previous prophylaxis with aerosolized pentamidine, and simultaneous antiretroviral treatment were allowed in the study protocol. Exclusion criteria were prior toxoplasmic encephalitis, concurrent treatment with drugs active against $P$. carinii or $T$. gondii, pregnancy, injection drug use, and a serum creatinine level of $>120 \mu \mathrm{mol} / \mathrm{L}$. The study design was approved by the ethical committees of the participating hospitals, and all participants gave informed consent.

\section{Study Design}

This controlled, open-label trial was conducted in 7 centers in Switzerland, 4 centers in Milan, Italy, and 1 center in Besançon, France. Enrollment began in February 1991. Participants were randomized to receive either $200 \mathrm{mg}$ of dapsone and $75 \mathrm{mg}$ of pyrimethamine once weekly or 300 $\mathrm{mg}$ of aerosolized pentamidine every 4 weeks, by means of a Respirgard II jet nebulizer (Marquest, Englewood, $\mathrm{CO}$ ) and under the supervision of a nurse. Block randomization was used, with additional stratification by center, previous PCP, and positivity or negativity for toxoplasma IgG, as determined by ELISA. Folinic acid was not routinely administered. On the basis of the observation that failures of pentamidine prophylaxis tend to occur early [2], participants who had not previously received aerosolized pentamidine were given a loading dose of three inhalations of $300 \mathrm{mg}$ each within 10-14 days; monthly inhalations took place thereafter.

The protocol allowed a change of treatment in case of intolerance or the occurrence of breakthrough PCP. As of October 1991, a high rate of treatment switches from dapsone/pyrimethamine to aerosolized pentamidine necessitated a change of protocol: each study candidate first re- ceived three trial doses of dapsone/pyrimethamine (200/75 $\mathrm{mg}$ once a week), and only those patients who tolerated these doses were enrolled. A 2:1 randomization ratio in favor of dapsone/pyrimethamine was additionally introduced to compensate for the treatment switches.

\section{Supply and Administration of Dapsone/Pyrimethamine}

Because both dapsone and pyrimethamine were available in Switzerland only as $25-\mathrm{mg}$ tablets, the generic drugs were assembled into one capsule containing $200 \mathrm{mg}$ of dapsone and $75 \mathrm{mg}$ of pyrimethamine (G. Streuli and Co., Uznach, Switzerland), thus enabling the participants to take only one capsule once weekly. The capsules were analyzed and found to contain, on average, $196 \mathrm{mg}$ of dapsone and $75.5 \mathrm{mg}$ of pyrimethamine; the weight uniformity was within $10 \%$ for all capsules examined. A pharmacokinetic evaluation of the combination capsule involving three volunteers demonstrated normal bioavailability in comparison to that of commercially available tablets [20]. The combination capsules were purchased for an average price of $\$ 0.15$ each. The patients were instructed to take dapsone/pyrimethamine separate from didanosine to avoid any possible interaction between the two treatments that might decrease dapsone efficacy [21].

\section{Follow-up of Patients and Clinical End Points}

The participants were observed every 4 weeks and evaluated for symptoms and adverse events as well as tested for hematologic, renal, and hepatic function. During every visit, patients were asked about their compliance and the number of missed capsules was noted. An algorithm concerning the management of adverse events was provided, allowing for treatment switches (crossovers) in cases of moderate to severe clinical symptoms clearly attributable to the study medication or in cases of persisting, severe hematologic toxicity.

Diagnoses of PCP or toxoplasmic encephalitis were considered as definite if the presence of $P$. carinii or $T$. gondii was microscopically confirmed. A diagnosis of presumed PCP was made according to World Health Organization criteria [22] and required all of the following: dyspnea on exertion or nonproductive cough of recent onset; diffuse interstitial infiltrates evident on a chest roentgenogram; hypoxemia, with arterial partial pressure of oxygen $\left(\mathrm{pO}_{2}\right)$ of $<70 \mathrm{mmHg}$ or an increased arterial-alveolar oxygen difference; and no evidence of bacterial pneumonia or of another condition that would account for the symptoms. Because a brain biopsy is not a routine diagnostic approach [11], most of the diagnoses of toxoplasmic encephalitis were presumed on the basis of all of the following: recent onset of a focal neurological abnormality consistent with intracranial disease or a reduced level of consciousness, evidence of intracranial lesion(s) on computed tomography or magnetic resonance imaging, and posi- 
tivity for serum antibodies to toxoplasmosis or successful response to antitoxoplasmic therapy [22].

\section{Statistical Analysis}

Primary end points were the occurrences of definite or presumed PCP or toxoplasmic encephalitis. The statistical analyses were based on the intent-to-treat principle and included events occurring until 31 December 1992. Where specified, occurrence of PCP or toxoplasmic encephalitis during the originally assigned treatment was also analyzed. For this purpose, the duration was equal to the time of initial treatment, and only those events that occurred during the treatment or within 1 month of its discontinuation were considered. Two patients who never started receiving the prophylaxis were excluded from the analysis. Baseline characteristics were compared with use of the $\chi^{2}$ test or Mann-Whitney test, as appropriate. The probability of remaining free of disease was calculated by means of Kaplan-Meier life-table analysis and compared by log-rank test. Multivariate Cox proportional hazards regression was used to estimate relative risks associated with the regimens; the models were adjusted for CD4 lymphocyte counts at baseline and additionally for toxoplasma serology when the risk of toxoplasmic encephalitis was evaluated.

Four interim analyses were planned, and rules for stopping the trial were based on those of O'Brien and Fleming [23]. The third interim analysis in December 1992 revealed no significant differences in the intent-to-treat analysis. At this time, however, it became clear that the latter lost power because of the frequent intolerance of dapsone/ pyrimethamine and subsequent switches to aerosolized pentamidine. In addition, new data suggested that dapsone/ pyrimethamine [10] and possibly trimethoprim-sulfamethoxazole [24] prevent toxoplasmosis. Meanwhile, administration of trimethoprim-sulfamethoxazole became the standard prophylaxis for PCP $[5,6,25]$, and continuing the prophylactic administration of aerosolized pentamidine to patients who were seropositive for toxoplasmosis seemed unethical. The trial was therefore stopped prematurely as of 31 December 1992.

Because the substantial number of switches from dapsone/ pyrimethamine to aerosolized pentamidine diminished the power of the intent-to-treat analysis to detect a difference between the effects of treatment [26], a subgroup analysis was subsequently performed to evaluate the biological effect of dapsone/pyrimethamine. One group consisted of all participants randomized to receive dapsone/pyrimethamine who did not need to withdraw from the treatment because of intolerance and who had received no previous trial dose $(n=$ $120)$. Of the 182 participants randomized to receive aerosolized pentamidine and who fulfilled the same criteria, 120 were matched to the dapsone/pyrimethamine group members according to their toxoplasma serology, their CD4 count (within 10 cells $/ \mu \mathrm{L}$ in the $<200 / \mu \mathrm{L}$ range, within $\pm 10 \%$ in the $>200 / \mu \mathrm{L}$ range, and within $\pm 20 \%$ in the $>350 /$ $\mu \mathrm{L}$ range), and (when possible) their sex and date of randomization. The same end points were used as in the intent-totreat analysis. Both groups were then compared for occurrence of PCP, toxoplasmic encephalitis, and death (logrank test and multivariate Cox regression analysis).

\section{Results}

\section{Patients' Characteristics}

A total of 533 patients started the treatment; 382 were randomized before the institution of trial dosing on 1 October 1991, and 151 had tolerated the three (weekly) trial doses of dapsone/pyrimethamine and were randomized thereafter at a ratio of 2:1 favoring the dapsone/ pyrimethamine regimen. Overall, 291 participants were randomized to receive dapsone/pyrimethamine and 242 to receive aerosolized pentamidine. Participants continued to be monitored even if they prematurely discontinued taking the study medication because of (1) opportunistic diseases or treatment with drugs not permitted in the protocol $(n=56)$, (2) patient-related reasons such as noncompliance or withdrawal of consent $(n=97)$, (3) intolerance after switching to the other treatment $(n=1)$, or $(4)$ other reasons $(n=4)$. As of 31 December 1992, 255 participants were still receiving therapy (112, dapsone/pyrimethamine; 143, aerosolized pentamidine), 150 had died, and 27 had been lost to followup for $>2$ months. The median duration of follow-up was 483 days (449 days in the dapsone/pyrimethamine group and 505 days in the aerosolized pentamidine group), and the total observation times were 4,113 patient-months (dapsone/pyrimethamine) and 3,659 patient-months (aerosolized pentamidine), based on the original treatment assignment.

The baseline characteristics of the two groups were well balanced (table 1). The median age was 33.5 years, and $83 \%$ of the patients were male. Only $19 \%$ had previously had PCP and were thus receiving secondary PCP prophylaxis. The seroprevalence of toxoplasma IgG was $47.5 \%$. Significant differences between the groups were observed only regarding the risk for HIV infection and prior prophylaxis with aerosolized pentamidine.

\section{Occurrence of PCP}

A total of 25 cases of PCP were observed, 19 in the 430 participants receiving primary prophylaxis and 6 in the 103 patients receiving secondary prophylaxis. The diagnosis of PCP was confirmed microscopically in 24 cases and was presumptive in one case. PCP occurred in 12 participants randomized to receive dapsone/pyrimethamine and in $13 \mathrm{al}-$ located to receive aerosolized pentamidine in the intent-to- 
Table 1. Baseline characteristics of HIV-infected patients who received dapsone/pyrimethamine (DP) or aerosolized pentamidine (AP) as prophylaxis for PCP and toxoplasmic encephalitis.

\begin{tabular}{|c|c|c|c|}
\hline \multirow[b]{2}{*}{ Characteristic } & \multicolumn{2}{|c|}{ Prophylaxis group } & \multirow[b]{2}{*}{$P$ value } \\
\hline & $\begin{array}{c}\text { DP } \\
(n=291)\end{array}$ & $\begin{array}{c}\text { AP } \\
(n=242)\end{array}$ & \\
\hline Median age $(y)$ & 34 & 33 & .28 \\
\hline Male sex (\%) & 84 & 83 & .63 \\
\hline Homosexual or bisexual (\%) & 43 & 34 & .04 \\
\hline Injection drug use (\%) & 34 & 41 & .11 \\
\hline Previous PCP (\%) & 18 & 21 & .48 \\
\hline Previous use of AP (\%) & 26 & 42 & $<.001$ \\
\hline $\begin{array}{l}\text { Previous use of zidovudine or } \\
\text { didanosine (\%) }\end{array}$ & 90 & 91 & .73 \\
\hline $\begin{array}{l}\text { Median CD4 lymphocyte count } \\
\quad(\text { per } \mu \mathrm{L})\end{array}$ & 116 & 105 & .30 \\
\hline Positivity for toxoplasma IgG (\%) & 46 & 49 & .59 \\
\hline
\end{tabular}

treat analysis (figure $1 ; P=.65, \log$-rank test). The adjusted relative risk was 0.89 (95\% confidence interval [CI], 0.39$1.89 ; P=.71$ ) for patients randomized to the dapsone/ pyrimethamine group in comparison with that for those randomized to the aerosolized pentamidine group.

Three of 12 cases of PCP in the dapsone/pyrimethamine group occurred between 184 and 414 days after a switch to aerosolized pentamidine due to intolerance. An additional two patients in each group developed PCP at least I month after all treatment had been discontinued (table 2). The inci-

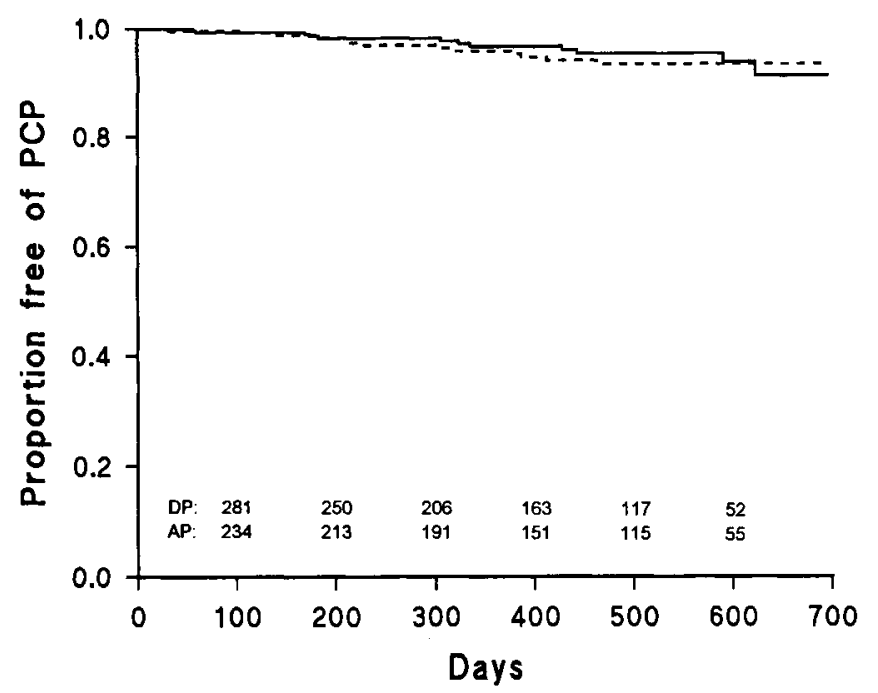

Figure 1. Proportion of participants remaining free of $P C P$ in an intent-to-treat analysis. The adjusted relative risk of PCP was 0.87 (95\% CI, 0.39-1.90) for those randomized to receive prophylaxis with dapsone/pyrimethamine. Numbers of patients at risk are listed above the time axis; DP = dapsone/pyrimethamine treatment group (solid line), and AP = aerosolized pentamidine treatment group (dashed line).
Table 2. Duration of follow-up and occurrence of clinical events in primary and secondary analyses of participants in the dapsone/ pyrimethamine (DP) and aerosolized pentamidine (AP) prophylaxis groups.

\begin{tabular}{|c|c|c|c|}
\hline \multirow[b]{2}{*}{ Variable } & \multicolumn{2}{|c|}{$\begin{array}{l}\text { Prophylaxis } \\
\text { group }\end{array}$} & \multirow[b]{2}{*}{$P$ value } \\
\hline & $\mathrm{DP}$ & AP & \\
\hline \multicolumn{4}{|l|}{ Intent-to-treat analysis } \\
\hline Median follow-up (d) & 449 & 505 & \\
\hline \multicolumn{4}{|l|}{ Clinical event (no. of patients) } \\
\hline PCP & 12 & 13 & .65 \\
\hline Toxoplasmic encephalitis & 14 & 20 & .17 \\
\hline Death & 77 & 73 & .93 \\
\hline \multicolumn{4}{|l|}{ As-treated analysis* } \\
\hline Median follow-up (d) & 226 & 379 & \\
\hline \multicolumn{4}{|l|}{ Clinical event (no. of patients) } \\
\hline PCP & 7 & 11 & .63 \\
\hline Toxoplasmic encephalitis & 2 & 17 & $<.005$ \\
\hline Death & 29 & 40 & .84 \\
\hline \multicolumn{4}{|l|}{ Analysis of biological effect ${ }^{\dagger}$} \\
\hline Median follow-up (d) & 537 & 549 & \\
\hline \multicolumn{4}{|l|}{ Clinical event (no. of patients) } \\
\hline $\mathrm{PCP}$ & 7 & 5 & .47 \\
\hline Toxoplasmic encephalitis & 3 & 14 & $<.01$ \\
\hline Death & 40 & 41 & .82 \\
\hline
\end{tabular}

NOTE. $P$ values are based on log-rank test results. Only two patients who had PCP while taking dapsone/pyrimethamine and none who developed toxoplasmic encephalitis were concomitantly receiving didanosine. None of the cases of breakthrough PCP or toxoplasmic encephalitis that occurred during the treatment were associated with poor compliance.

* Analysis of events occurring during initially assigned treatment (or within 30 days of its discontinuation).

${ }^{\dagger}$ Subgroup analysis of participants who had no prior trial dose and tolerated dapsone/pyrimethamine $(n=120)$ vs. matched group tolerating aerosolized pentamidine $(n=120)$. The groups were matched in regard to toxoplasma serology, CD4 lymphocyte count, and (when possible) sex and date of randomization

dence of PCP per 100 patient-years during the initial treatment was thus 3.37 (95\% CI. 1.33-6.32) among dapsone/ pyrimethamine recipients and 4.48 (95\% CI, 2.22-7.52) among aerosolized pentamidine recipients, corresponding to an adjusted relative risk of $0.96(95 \% \mathrm{CI}, 0.37-2.51 ; P=.93)$ for patients taking dapsone/pyrimethamine.

PCP developed in 6 patients receiving secondary prophylaxis, 1 in the dapsone/pyrimethamine group and 5 in the aerosolized pentamidine group, all of whom were receiving the originally assigned treatment. In the Kaplan-Meier analysis, the proportion free of PCP at 1 year was $0.96(95 \% \mathrm{Cl}$, $0.93-0.98)$ of the dapsone/pyrimethamine recipients vs. 0.97 (95\% CI, 0.92-0.98) of the aerosolized pentamidine recipients undergoing primary prophylaxis and 0.98 (95\% CI, $0.96-1.00)$ vs. 0.93 (95\% CI, $0.80-0.98)$, respectively, of those undergoing secondary prophylaxis.

The median time to onset of PCP was 315 days (range, 56-622 days) for those randomized to receive dapsone/ 
pyrimethamine and 224 days (range, 30-463 days) for those receiving aerosolized pentamidine. The median $\mathrm{CD} 4$ count at the time of randomization was $51 / \mu \mathrm{L}$ (range, $6-370 / \mu \mathrm{L}$ ) in those patients who eventually developed PCP; $84 \%$ of them had CD4 lymphocyte counts of $<200 / \mu \mathrm{L}$. The CD4 counts of the remaining four patients ranged between $200 /$ $\mu \mathrm{L}$ and $370 / \mu \mathrm{L}$ (22\%-26\% of total lymphocytes) at randomization. One patient receiving aerosolized pentamidine was found to have extrapulmonary $P$. carinii infection of the spleen and liver and was not included in this analysis. Four patients died of PCP within 3 weeks of the diagnosis; three of them had been receiving aerosolized pentamidine and one dapsone/pyrimethamine.

\section{Occurrence of Toxoplasmic Encephalitis}

Toxoplasmic encephalitis developed in 34 patients during the study. In the intent-to-treat analysis, 14 cases occurred in the dapsone/pyrimethamine group and 20 in the aerosolized pentamidine group $(P=.17, \log$-rank test), corresponding to an adjusted relative risk of $0.56(\mathrm{CI}, 0.28-1.12 ; P=.10)$ for patients randomized to receive dapsone/pyrimethamine. At the time of diagnosis, however, 12 of 14 patients with toxoplasmic encephalitis had not been receiving dapsone/pyrimethamine for a median of 172 days (range, 34-421 days). Eleven of these 12 patients had previously switched to aerosolized pentamidine because of intolerance of dapsone/ pyrimethamine; two were not receiving any study medication at all (table 2).

While they were undergoing the assigned treatment, toxoplasmic encephalitis developed in two dapsone/ pyrimethamine recipients and 17 aerosolized pentamidine recipients, corresponding to incidence rates of 0.96 (95\% CI, 0.09-2.76) and 6.92 (95\% CI, 4.02-10.6) per 100 patient-years in the respective groups. The adjusted relative risk of developing toxoplasmic encephalitis during treatment was $0.14(95 \% \mathrm{CI}, 0.03-0.61 ; P<.01)$ for patients taking dapsone/pyrimethamine compared to that for those taking aerosolized pentamidine.

The diagnosis was presumptive in 26 cases and definite in eight cases. Among the presumptive cases, only two were diagnosed on the basis of a positive serology despite lack of response to specific therapy; in all others there was a response to antitoxoplasmic treatment. At the time of randomization, 31 (91\%) of 34 patients in whom toxoplasmic encephalitis eventually developed were seropositive for IgG antibodies to Toxoplasma species. One of the three seronegative patients was retested at the time of diagnosis and again had no antibodies detected by ELISA but responded promptly to antitoxoplasmic therapy. The two other patients were not retested; in one of them, however, disseminated toxoplasmosis was confirmed by autopsy. Three patients died of toxoplasmic encephalitis within 6 weeks after the diagnosis. Two cases diagnosed
Table 3. Probability of development of toxoplasmic encephalitis in patients for whom toxoplasma serology was positive and who were randomized to receive aerosolized pentamidine.

\begin{tabular}{lccc}
\hline & \multicolumn{3}{c}{$\begin{array}{c}\text { Probability* at indicated point. } \\
\text { post-randomization }\end{array}$} \\
\cline { 2 - 4 } $\begin{array}{l}\text { Initial CD4 lymphocyte } \\
\text { count }(n)\end{array}$ & 6 mo & 12 mo & 18 mo \\
\hline $0-199 / \mu \mathrm{L}(90)$ & $7(3-15)$ & $13(7-23)$ & $23(14-36)$ \\
$0-99 / \mu \mathrm{L}(49)$ & $13(6-28)$ & $20(10-37)$ & $35(20-56)$ \\
$100-199 / \mu \mathrm{L}(41)$ & $0(0-0)$ & $6(1-21)$ & $12(5-29)$ \\
\hline
\end{tabular}

* Percentage of probability (Kaplan-Meier estimates); $95 \%$ confidence interval is in parentheses.

at autopsy were of disseminated toxoplasmosis; myocarditis occurred in both cases, and involvement of the lung, liver, and spleen occurred in one.

The median time from randomization to onset of toxoplasmosis was 278 days (range, 30-634 days) and was similar in the two prophylaxis groups. The median CD4 count at the time of randomization was $61 / \mu \mathrm{L}$ (range, $3-218 / \mu \mathrm{L}$ ) for those patients in whom toxoplasmic encephalitis subsequently developed; $94 \%$ of them had a CD4 lymphocytes count of $<200 / \mu \mathrm{L}$, and for $71 \%$ it was $<100 / \mu \mathrm{L}$. Two patients who subsequently had toxoplasmosis had initial CD 4 counts of $200 / \mu \mathrm{L}$ ( $7 \%$ of total lymphocytes) and $218 / \mu \mathrm{L}$ (13\% of total lymphocytes), respectively. The risk to develop toxoplasmic encephalitis in seropositive patients without prophylaxis is summarized in table 3.

\section{Mortality}

One-hundred fifty participants died during the study, 77 of whom were randomized to receive dapsone/pyrimethamine and 73 to receive aerosolized pentamidine. The mortality was identical in both groups, with an adjusted relative risk of 1.01 (95\% CI, $0.74-1.40 ; P=.93$ ) for those randomized to receive dapsone/pyrimethamine. Sixty-nine patients died during initial treatment or within 30 days of its discontinuation: 29 received dapsone/pyrimethamine and 40 received aerosolized pentamidine, corresponding to an adjusted relative risk of $1.16(95 \% \mathrm{CI}, 0.72-1.89 ; P=.54)$ for recipients of dapsone/pyrimethamine.

\section{Subgroup Analysis of the Biological Effect of Dapsone/ Pyrimethamine}

To eliminate the interference of intolerance and crossovers between treatments, a subanalysis of two matched groups was performed. For every patient randomized to receive dapsone/pyrimethamine who did not need to stop the treatment because of intolerance, a matching patient within the aerosolized pentamidine group was selected. Matching 
was done in regard to toxoplasma serology and CD4 lymphocyte count only among patients who had not been given prior test doses; as a result, there were two groups of 120 patients with comparable baseline values (median CD4 lymphocyte counts, $134 / \mu \mathrm{L}$ for the dapsone/pyrimethamine recipients vs. $140 / \mu \mathrm{L}$ for the aerosolized pentamidine recipients) and a similar length of follow-up (537 and 546 days, respectively). The same end points were used as in the intent-to-treat analysis. The adjusted relative risk for those randomized to receive dapsone/pyrimethamine compared with that for those randomized to receive aerosolized pentamidine was $1.17(95 \% \mathrm{CI}, 0.76-1.81 ; P=.48)$ for death, 1.62 (95\% CI, 0.51-5.13; $P=.41$ ) for occurrence of PCP, and 0.21 (95\% CI, 0.06-0.72;P=.014) for occurrence of toxoplasmic encephalitis (figure 2).

\section{Intolerance}

Thirty percent of patients assigned to dapsone/ pyrimethamine prophylaxis switched to aerosolized pentamidine because of intolerance. The reported reasons for switching were nausea $(51 \%)$, skin rash $(40 \%)$, headache (37\%), fever (34\%), anemia (29\%), anorexia (17\%), leukopenia $(7 \%)$, pancytopenia $(6 \%)$, vomiting $(6 \%)$, diarrhea $(3 \%)$, and hepatitis (1\%). The adverse events were reversible, and no severe cutaneous reactions were reported. Before the introduction of the trial doses, $38 \%$ of patients receiving dapsone/pyrimethamine switched because of intolerance. After the routine administration of three trial doses before the randomization, 33 (18\%) of 184 study candidates were intolerant of the treatment and were not enrolled in the trial; the remaining $82 \%$ were randomized. Fifteen percent of these patients subsequently switched treatment because of eventual intolerance. In a multivariate Cox proportional hazard regression analysis of 87 participants who discontinued dapsone/pyrimethamine prophylaxis because of intolerance vs. 204 participants who tolerated dapsone/pyrimethamine, the following factors were independently predictive of a treatment switch due to intolerance: no previous trial dose, relative risk of $2.90(95 \% \mathrm{Cl}, 1.65-5.10 ; P<.001)$; female sex, relative risk of $1.87(95 \% \mathrm{CI}, 1.11-3.13 ; P<.02)$; and low CD4 lymphocyte count, relative risk of $1.66(95 \% \mathrm{CI}, 1.30-$ $2.13 ; P<.001$ ) for each decrease by $100 / \mu \mathrm{L}$. The rate of discontinuation of dapsone/pyrimethamine prophylaxis was highest during the first 3-4 months of treatment, but the proportion of participants remaining in the treatment group continued to drop throughout the entire first year (figure 3). Four percent of participants receiving aerosolized pentamidine discontinued the treatment because of bronchospasm, cough, vomiting, claustrophobia, or bitter taste in the mouth.

The laboratory values did not differ between the two prophylaxis groups at the time of randomization. During the assigned treatment, the only significant differences in laboratory-defined toxic effects involved a more prominent de-
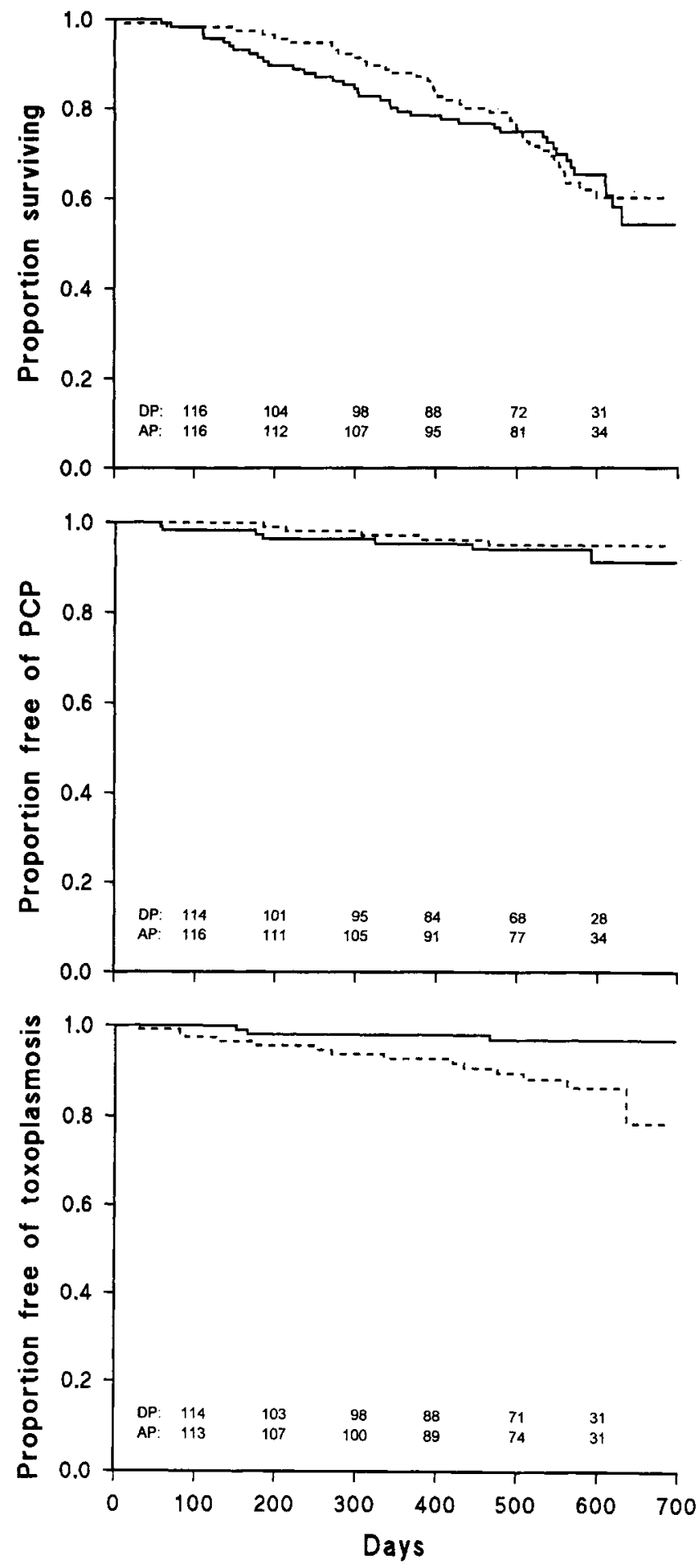

Figure 2. Analysis of the biological effect in 120 participants who tolerated dapsone/pyrimethamine and 120 matched participants in the aerosolized pentamidine treatment group. The adjusted relative risks for patients randomized to receive dapsone/ pyrimethamine were $1.17(95 \% \mathrm{CI}, 0.76-1.81)$ for occurrence of death (top panel), 1.62 (95\% CI, 0.5 I-5.13) for occurrence of PCP (middle panel), and $0.21(95 \% \mathrm{CI}, 0.06-0.72 ; P=.014)$ for occurrence of toxoplasmic encephalitis (bottom panel). Numbers of patients at risk are listed above the time axes; DP = dapsone/ pyrimethamine treatment group (solid line), and AP = aerosolized pentamidine treatment group (dashed line). 


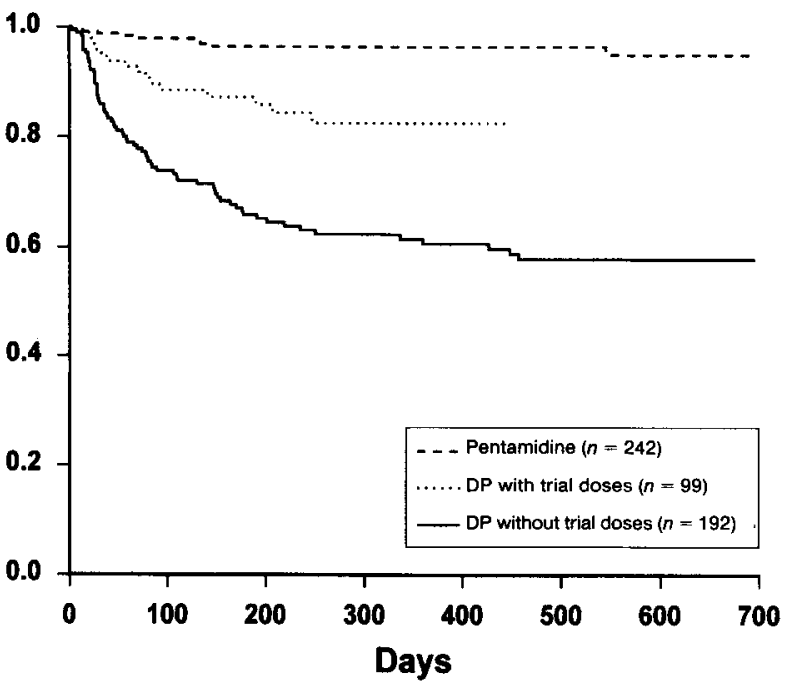

Figure 3. Proportion of participants still receiving initially assigned treatment during course of study. The rate at which treatment was stopped because of intolerance of dapsone/ pyrimethamine (DP) was higher among patients who did not receive the three trial doses ( 72 of 192) than among those who had received and tolerated them before randomization ( 15 of 99). The differences among the groups were significant $(P<.001$, log-rank test).

crease in hemoglobin $(-1.8 \mathrm{~g} / \mathrm{dL}$ vs. $-1.4 \mathrm{~g} / \mathrm{dL} ; P<.01)$ and a higher increase in methemoglobin $(+0.7 \%$ vs. $+0.3 \%$ of hemoglobin; $P<.02)$ in dapsone/pyrimethamine recipients. The percentage of participants in whom grade 3 or 4 toxicity developed did not differ significantly; neutropenia (neutrophil count, $<0.75 \times 10^{9} / \mathrm{L}$ ) was observed in $16.3 \%$ of dapsone/pyrimethamine recipients and in $17.1 \%$ of aerosolized pentamidine recipients, and severe anemia (hemoglobin, $<8.0 \mathrm{~g} / \mathrm{dL})$ in $9.2 \%$ and $5.4 \%$, respectively $(P=.11)$. Folinic acid was rarely administered but was more frequently given to recipients of dapsone/pyrimethamine $(7.2 \%$ vs. $3.3 \%$ of participants received it at least once during the study; $P$ $<.05)$.

\section{Discussion}

In this randomized, open-label trial, dapsone/pyrimethamine and aerosolized pentamidine were equally effective as primary and secondary prophylaxis for PCP. The efficacy of dapsone/pyrimethamine as primary prophylaxis for toxoplasmic encephalitis was not significant in an intent-to-treat analysis (adjusted relative risk, $0.56 ; P=.10$ ). However, 12 of 14 cases of toxoplasmic encephalitis in participants randomized to receive dapsone/pyrimethamine occurred a median of 6 months after the treatment had been discontinued. Frequent symptoms of intolerance of dapsone/pyrimethamine caused $30 \%$ of participants to cross over to prophylaxis with aerosolized pentamidine, whereas only $4 \%$ crossed over to prophylaxis with dapsone/pyrimethamine. Because of this substantial number of treatment switches, the power of the intent-to-treat analysis to detect a difference between the efficacy of treatments was reduced [26], and the results are difficult to interpret. If the analysis is restricted to those events that occurred during the originally assigned treatment (or within 1 month after its discontinuation), the reduction in the incidence of toxoplasmic encephalitis among recipients of dapsone/pyrimethamine is dramatic (table 2).

Participants who prematurely discontinued dapsone/ pyrimethamine prophylaxis because of intolerance had fewer CD4 lymphocytes. Low CD4 counts are correlated with an increased risk of toxoplasmosis [9-11], and the lack of follow-up of intolerant patients after treatment stopped might therefore have biased the results in favor of dapsone/ pyrimethamine in the as-treated analysis [27]. An analysis of the biological effect of dapsone/pyrimethamine was therefore performed involving patients who tolerated the treatment and who were matched to aerosolized pentamidine recipients in regard to toxoplasma serology and CD4 lymphocyte count. The comparison of these two groups, similar in terms of prognostic factors, yielded evidence of a significantly reduced incidence of toxoplasmic encephalitis among participants randomized to receive dapsone/pyrimethamine (relative risk, $0.21 ; P=.014$ ), a finding confirming the antitoxoplasmic efficacy of the prophylaxis in those patients who could tolerate it (biological effect).

Once-weekly administration of both dapsone and pyrimethamine proved to be an effective and easy regimen. As expected on the basis of the half-lives of 23-25 hours for dapsone and 80-105 hours for pyrimethamine [28, 29], serum concentrations of dapsone decreased considerably throughout the weekly dosing interval, whereas pyrimethamine levels remained relatively stable in most of the sera examined [20]. Maintenance of high serum levels may be less important for prophylaxis than for cure, as shown by the experience with weekly doses of dapsone [18] and that with intermittent doses of trimethoprim-sulfamethoxazole [6, 24 , 30]: both regimens prevented PCP, although the dosing interval exceeded the half-life of the individual compounds severalfold. Investigators in one of the latter studies reported undetectable trough levels in the majority of patients despite prophylactic efficacy [30].

Fifty $\mathrm{mg}$ of dapsone daily and $50 \mathrm{mg}$ of pyrimethamine once a week were effective as primary prophylaxis for PCP and toxoplasmic encephalitis in a previous trial [10]. In contrast, $100 \mathrm{mg}$ of dapsone and $25 \mathrm{mg}$ of pyrimethamine once weekly are probably not enough to prevent either PCP or toxoplasmic encephalitis [31, 32], although the number of cases in these prospective studies was low. Total weekly doses of 200-300 mg of dapsone and 50-75 mg of pyrimethamine thus seem to be necessary for protection against both opportunistic diseases.

Dapsone/pyrimethamine remains the best-studied combination with proven prophylactic efficacy against toxoplasmic 
encephalitis. Moreover, it is the cheapest available; the monthly cost of once weekly administration is $<\$ 1$. Dapsone alone (100 mg twice weekly) may also be effective as primary prophylaxis for toxoplasmic encephalitis [33], but the statistical analysis of this trial was not conclusive $(P=.07, \log -$ rank test) and the percentage of seropositive patients was unknown.

Prophylaxis with trimethoprim-sulfamethoxazole also appears to protect against toxoplasmic encephalitis, but the optimal dose is unknown and the assessments of efficacy have been based only on a retrospective analysis [24], observational data $[34,35]$, or results of controlled trials that did not primarily target prophylaxis for toxoplasmic encephalitis and therefore yielded small numbers of events [5, 6, 31, 36-38]. While none of these trials revealed a significant protective effect on an intent-to-treat basis, toxoplasmic encephalitis in fact rarely occurred during treatment with trimethoprim-sulfamethoxazole. Nevertheless, the efficacy of trimethoprimsulfamethoxazole as primary prophylaxis for toxoplasmic encephalitis remains to be proved in a prospective, controlled study. Pyrimethamine/sulfadoxine has also been used as prophylaxis for PCP and toxoplasmic encephalitis, but the combination has not been tested in controlled prospective trials and cannot be generally recommended.

The development of toxoplasmic encephalitis in HIV-infected patients represents the reactivation of a previous, silent infection in $>95 \%$ of all cases [10-13]. Among individuals with positive toxoplasma serology, toxoplasmic encephalitis accounts for $24 \%-38 \%$ of all AIDS-defining diagnoses $[12,13]$. In patients with $\mathrm{CD} 4$ counts of $<100 / \mu \mathrm{L}$ for whom toxoplasma serology is positive and who have received no prophylaxis, the risk of reactivation rises to $20 \%$ $34 \%$ after 12 months and to $35 \%-40 \%$ after 18 months, according to our study (table 3 ) and other prospective studies $[10,39]$. Patients with positive toxoplasma serology and CD4 counts of $<100 / \mu \mathrm{L}$ should therefore be mainly targeted to receive primary prophylaxis. However, only $68 \%$ of all cases of toxoplasmic encephalitis in our study occurred in patients with these characteristics at the time of randomization, a finding illustrating that a smaller yet not negligible risk also exists in association with a CD4 range of 100-200/ $\mu \mathrm{L}$. The risk of toxoplasmosis in a population depends on the prevalence of positive toxoplasma serology, which varies from $14 \%$ to $35 \%$ in the United States [40] and from $13 \%$ to $23 \%$ in the United Kingdom $[41,42]$ and rise to $50 \%$ in Switzerland [14] and up to $>70 \%$ in France [43]. In addition, seroprevalence rises with age within a given population $[14,42]$.

For PCP prophylaxis, trimethoprim-sulfamethoxazole is more effective than aerosolized pentamidine $[5,6]$, and it became the first-line prophylactic agent in 1992 [25]. Weekly doses of 3-7 double-strength tablets of trimethoprim-sulfamethoxazole appear equally effective [6]. The percentage of patients discontinuing the treatment because of intolerance varied between $9 \%$ and $31 \%$ in prospective trials $[5,6,30,36,38,44]$. The intolerance was not clearly dosedependent, although newer results indicate that daily administration of one double-strength table causes earlier and greater toxic effects than does thrice weekly administration $[6,38,45]$.

Oral dapsone is an alternative prophylactic agent for PCP, although it may be slightly less effective than trimethoprimsulfamethoxazole. Dapsone at a dosage of $100 \mathrm{mg}$ twice weekly was as effective as aerosolized pentamidine in two comparative studies $[33,46]$, and at a dosage of $100 \mathrm{mg}$ daily it was as effective as trimethoprim-sulfamethoxazole [47]. In a recently completed large randomized trial, overall results with dapsone at a dosage of $100 \mathrm{mg}$ daily were comparable to those with aerosolized pentamidine and trimethoprim-sulfamethoxazole; however, dapsone appeared less biologically active than the latter drug (Dr. S. A. Bozzette, personal communication). The combination dapsone/pyrimethamine (350/50 mg per week) was recently shown to be as effective as aerosolized pentamidine as primary PCP prophylaxis [10]. The efficacy of lower dosages of dapsone/pyrimethamine (100/25 mg once to twice weekly) was similar to that of trimethoprim-sulfamethoxazole and of aerosolized pentamidine in one study [31] but was inferior to that of trimethoprim-sulfamethoxazole in other prospective studies [32, 44]. The reported rates of discontinuation of therapy due to intolerance were $2 \%-5 \%, 11 \%-12 \%, 24 \%-35 \%$, and $43 \%$ for weekly dapsone doses of $100 \mathrm{mg}[31,32], 200 \mathrm{mg}$ [33, 44, 46], $350 \mathrm{mg}[10,48]$, and $700 \mathrm{mg}$ [47], respectively. Intolerance of dapsone with or without pyrimethamine thus seems to be dose-dependent and only slightly higher than that of trimethoprim-sulfamethoxazole, on the basis of prospective data. As $55 \%-60 \%$ of patients intolerant of trimethoprim-sulfamethoxazole will be able to tolerate dapsone $[47,49]$, the option to cross over to an alternative medication should be given to individuals not tolerating trimethoprim-sulfamethoxazole.

Tolerance of dapsone/pyrimethamine in our study was poor although not much different from that noted in other trials of systemic prophylaxis $[5,10]$. Nausea, skin rash, headache, fever, and anemia were the most frequent reasons for discontinuation of the treatment. Hypersensitivity reactions are a well-recognized problem in the HIV-infected population, and on the basis of the toxicity profiles of dapsone and pyrimethamine, the reactions occurring in our study were most likely attributable to dapsone $[50,51]$. Hypersensitivity developed more frequently in participants with few CD4 lymphocytes, a circumstance that echoes the experience with amoxycillin-clavulanate [52] but is in contrast to a report associating hypersensitivity to trimethoprim-sulfamethoxazole with high CD4 counts [53]. The combination of dapsone/pyrimethamine had not been previously used in any of the study centers. As the rate of crossovers varied considerably between the study sites (between 19\% and 38\% in the 
large centers), the management of intolerance and the willingness to allow possibly temporary toxicity may not have been optimal early in the study.

The only significant laboratory-proven difference between the two prophylaxis groups in our study was a slightly higher incidence of anemia among dapsone/pyrimethamine recipients. Concomitant administration of dapsone and zidovudine can cause higher incidence of anemia, neutropenia, and thrombocytopenia [54], and the contribution of each of these agents to observed toxicity is difficult to assess. In contrast to the toxic effect of pyrimethamine on bone marrow, dapsone-induced hemolytic anemia is caused by the product of its cytochrome P450-mediated $N$-hydroxylation [55], which is not reversible by folinic acid. Because the hematologic toxic effects seen in our study were not greater in comparison with those associated with a similar regimen that was regularly supplemented with folinic acid [10], routine administration of folinic acid does not seem warranted during prophylactic treatment with dapsone/pyrimethamine at the dosage we used. Because the development of anemia is proportional to both concentration and time of exposure to dapsone hydroxylamine [55], slightly lower toxicity might have occurred with smaller doses of dapsone given more frequently. As the mortality rate was identical for both prophylaxis groups, data suggesting higher mortality among patients receiving prophylaxis with either dapsone [48] or pyrimethamine [56] are not supported by our study.

In conclusion, our data provide an additional basis on which to recommend primary prophylaxis for toxoplasmic encephalitis in patients who have positive toxoplasma serology and low CD4 counts, especially $<100 / \mu \mathrm{L}$. Prophylaxis with once-weekly dapsone/pyrimethamine is simple and cheap and prevents both PCP and toxoplasmic encephalitis in those patients who can tolerate it. The high rate of intolerance in patients with advanced immune deficiency stresses the need to evaluate better-tolerated prophylactic regimens.

\section{Acknowledgments}

The authors are indebted to all participants of the trial; to Karola Hodapp for data management; to Drs. Dominique Anwar (Lausanne), Rene Borgts (Bern), Jean-Philippe Chave (Lausanne), Urs Erlacher (Basel), Alessandro Gringeri (Centro Bianchi Bonomi IRCCS Policlinico, Milan), Peter Maurer (Basel), Raffaele Malinverni (Bern), Claudio Velati (Centro Trasfusionale, Ospedale di Bollate, Milan), Pietro Vernazza (St. Gallen), Maria C. Vivirito (Ia Div. Malattie Infettive, Ospedale Sacco, Milano), and Roberto Zerboni (Clinica Dermatologica IRCCS Policlinico, Milano) for management of the cases; and to Bernadette Mermillod and Drs. Theo Gasser and Steven N. Goodman for statistical advice.

\section{References}

1. Schwartländer B, Horsburgh CR Jr, Hamouda O, Skarabis H, Koch MA. Changes in the spectrum of AIDS-defining conditions and de- crease in CD4+ lymphocyte counts at AIDS manifestation in Germany from 1986 to 1991. AIDS 1992;6:413-20.

2. Hirschel B, Lazzarin A, Chopard P, et al. A controlled study of inhaled pentamidine for primary prevention of Pneumocystis carinii pneumonia. N Engl J Med 1991;324:1079-83.

3. Leoung GS, Feigal DW Jr, Montgomery AB, et al. Aerosolized pentamidine for prophylaxis against Pneumocystis carinii pneumonia: the San Francisco community prophylaxis trial. N Engl J Med 1990; 323:769-75.

4. Fischl MA, Dickinson GM, La Voie L. Safety and efficacy of sulfamethoxazole and trimethoprim chemoprophylaxis for Pneumocystis carinii pneumonia in AIDS. JAMA 1988;259:1185-9.

5. Hardy WD. Feinberg J, Finkelstein DM, et al. A controlled trial of trimethoprim-sulfamethoxazole or aerosolized pentamidine for secondary prophylaxis of Pneumocystis carinii pneumonia in patients with the acquired immunodeficiency syndrome. AIDS Clinical Trials Group Protocol 021. N Engl J Med 1992; 327:1842-8.

6. Schneider MME. Hoepelman AIM, Eeftinck Schattenkerk JKM, et al. A controlled trial of aerosolized pentamidine or trimethoprim-sulfamethoxazole as primary prophylaxis against Pneumocystis carinii pneumonia in patients with human immunodeficiency virus infection. N Engl J Med 1992; 327:1836-41.

7. Medina I, Mills J, Leoung G, et al. Oral therapy for Pneumoc ystis carinii pneumonia in the acquired immunodeficiency syndrome: a controlled trial of trimethoprim-sulfamethoxazole versus trimethoprimdapsone. N Engl J Med 1990; 323:776-82.

8. Kirby HB. Kenamore B. Guckian JC. Pneumoc ystis carinii pneumonia treated with pyrimethamine and sulfadiazine. Ann Intern Med 1971:75:505-9.

9. Heald A. Flepp M, Chave J-P. et al. Treatment for cerebral toxoplasmosis protects against Pneumocystis carinii pneumonia in patients with AIDS. Ann Intern Med 1991;115:760-3.

10. Girard P-M, Landman R. Gaudebout C, et al. Dapsone/pyrimethamine compared with aerosolized pentamidine as primary prophylaxis against Pneumocistis carinii pneumonia and toxoplasmosis in HIV infection. N Engl J Med 1993;328:1514-20.

11. Renold C, Sugar A. Chave J-P, et al. Toxoplasma encephalitis in patients with the acquired immunodeficiency syndrome. Medicine (Baltimore) 1992; 71:224-39

12. Grant IH. Gold JWM, Rosenblum M, Niedzwiecki D. Armstrong D. Toxoplasma gondii serology in HIV-infected patients: the development of central nervous system toxoplasmosis in AIDS. AIDS 1990; 4:519-21

13. Israelski DM, Chmiel JS, Poggensee L, Phair JP, Remington JS. Prevalence of toxoplasma infection in a cohort of homosexual men at risk of AIDS and toxoplasmic encephalitis. $J$ Acquir Immune Defic Syndr 1993;6:414-8.

14. Zufferey J. Sugar A. Rudaz P, Bille J, Glauser MP. Chave JP. Prevalence of latent toxoplasmosis and serological diagnosis of active infection in HIV-positive patients. Eur J Clin Microbiol Infect Dis $1993 ; 12: 591-5$.

15. Walzer PD. Foy J. Steele P, White M. Treatment of experimental pneumocystosis: review of 7 years of experience and development of a new system for classifying antimicrobial drugs. Antimicrob Agents Chemother 1992; 36:1943-50.

16. Allegra CJ, Boarman D. Kovacs JA, et al. Interaction of sulfonamide and sulfone compounds with Toxoplasma gondii dihydropteroate synthase. J Clin Invest 1990;85:371-9.

17. Derouin F, Piketty C, Chastang C, Chau F. Rouveix B, Pocidalo JJ. Anti-Toxoplasma effects of dapsone alone and combined with pyrimethamine. Antimicrob Agents Chemother 1991;35:252-5.

18. Hughes WT, Kennedy W, Dugdale M, et al. Prevention of Pneumocysis carinii pneumonitis in AIDS patients with weekly dapsone [letter]. Lancet 1990:336:1066. 
19. Phair J, Muñoz A, Detels R, et al. The risk of Pneumocystis carinii pneumonia among men infected with human immunodeficiency virus type I. N Engl J Med 1990;322:161-5.

20. Opravil M, Joos B, Lüthy $R$. Levels of dapsone and pyrimethamine in serum during once-weekly dosing for prophylaxis of Pneumocystis carinii pneumonia and toxoplasmic encephalitis. Antimicrob Agents Chemother 1994;38:1197-9.

21. Metroka CE, McMechan MF, Andrada R, Laubenstein LJ, Jacobus DP. Failure of prophylaxis with dapsone in patients taking dideoxyinosine [letter]. N Engl J Med 1991;325:737.

22. World Health Organization. Acquired immunodeficiency syndrome (AIDS): 1987 revision of CDC/WHO case definition for AIDS. Wkly Epidemiol Rec 1988;63:1-7.

23. O'Brien PC, Fleming TR. A multiple testing procedure for clinical trials. Biometrics 1979;35:549-56.

24. Carr A, Tindall B, Brew BJ, et al. Low-dose trimethoprim-sulfamethoxazole prophylaxis for toxoplasmic encephalitis in patients with AIDS. Ann Intern Med 1992; 1 17:106-11.

25. Centers for Disease Control. Recommendations for prophylaxis against Pneumocystis carinii pneumonia for adults and adolescents infected with human immunodeficiency virus. MMWR Morbid Mortal Wkly Rep 1992;4 I(RR 4): 1-11.

26. Lee YJ, Ellenberg JH, Hirtz DG, Nelson KB. Analysis of clinical trials by treatment actually received: is it really an option? Stat Med 1991; 10:1595-1605.

27. Lagakos SW, Lim LL-Y, Robins JM. Adjusting for early treatment termination in comparative clinical trials. Stat Med 1990;9:1417-24.

28. Edstein MD, Rieckmann KH, Veenendaal JR. Multiple-dose pharmacokinetics and in vitro antimalarial activity of dapsone plus pyrimethamine (Maloprim) in man. Br J Clin Pharmacol 1990; 30:25965.

29. Jones CR, Ovenell SM. Determination of plasma concentrations of dapsone, monoacetyl dapsone and pyrimethamine in human subjects dosed with maloprim. J Chromatogr 1979; 163:179-85.

30. Stein DS. Stevens RC. Terry D, et al. Use of low-dose trimethoprim-sulfamethoxazole thrice weekly for primary and secondary prophylaxis of Pneumocystis carinii pneumonia in human immunodeficiency virus-infected patients. Antimicrob Agents Chemother 1991; 35:1705-9.

31. Mallolas J, Zamora L. Gatell JM, et al. Primary prophylaxis for Pneumocystis carinii pneumonia: a randomized trial comparing cotrimoxazole. acrosolized pentamidine and dapsone plus pyrimethamine. AIDS 1993: 7:59-64.

32. Podzamczer D, Santin M, Jimenez J, Casanova A, Bolao F. Gudiol GRF. Thrice-weekly cotrimoxazole is better than weekly dapsone/ pyrimethamine for the primary prevention of Pneumocystis carinii pneumonia in HIV-infected patients. AIDS 1993; 7:501-6.

33. Torres RA. Barr M, Thorn M, et al. Randomized trial of dapsone and aerosolized pentamidine for the prophylaxis of Pneumocystis carinii pneumonia and toxoplasmic encephalitis. Am J Med 1993;95: 573-83.

34. Reynes $J$, Atoui $N$, Lassonnery $M$, et al. Co-trimoxazole in primary prophylaxis of cerebral toxoplasmosis in HIV-infected patients [abstract no P242]. In: Program and abstracts of the 3rd European Conference on Clinical Aspects and Treatment of HIV Infection. Paris: SIDA92. 1992:223.

35. Tournerie C, Charreau I. Cotrimoxazole for primary prophylaxis of toxoplasma encephalitis in advanced HIV patients [abstract no WAS-B 13-2]. In: Program and abstracts of the 9th International Conference on AIDS/IV STD World Congress (Berlin). Berlin: Institute for Clinical and Experimental Virology of the Free University of Berlin, 1993; I:56.

36. May T, Beuscart C, Reynes J, et al. Trimethoprim-sulfamethoxazole versus aerosolized pentamidine for primary prophylaxis of Pneumocystis carinii pneumonia: a prospective, randomized, controlled clinical trial. J Acquir Immune Defic Syndr 1994; 7:457-62.

37. Michelet C, Raffi F, Besnier JM, et al. Cotrimoxazole versus aerosolized pentamidine for primary prophylaxis of Pneumocystis carinii pneumonia [abstract no 1071]. In: Program and abstracts of the 33rd Interscience Conference on Antimicrobial Agents and Chemotherapy. Washington, DC: American Society for Microbiology, 1993:315.

38. Schneider MME, Nielsen TL, Hoepelman Al, et al. Two doses of trimethoprim-sulfamethoxazole as primary prophylaxis against $P$ neumocystis carinii pneumonia [abstract no 97]. In: Program and abstracts of the 33rd Interscience Conference on Antimicrobial Agents and Chemotherapy. Washington, DC: American Society for Microbiology, 1993:137.

39. Oksenhendler E, Charreau I, Tournerie C, Azihary M, Carbon C, Aboulker J-P. Toxoplasma gondii infection in advanced HIV infection. AlDS 1994;8:483-7.

40. Feldman HA. Epidemiology of toxoplasma infections. Epidemiol Rev 1982;4:204-13.

41. Gilbert RE, Tookey PA, Cubitt WD, Ades AE. Masters J, Peckham CS. Prevalence of toxoplasma $\operatorname{IgG}$ among pregnant women in west London according to country of birth and ethnic group. Br Med $\mathbf{J}$ 1993; 306: 185 .

42. Ades ARE, Nokes DJ. Modeling age- and time-specific incidence from seroprevalence: toxoplasmosis. Am J Epidemiol 1993;137: 1022-34.

43. Jeannel D. Niel G, Costagliola D, Danis M, Traore BM, Gentilini M. Epidemiology of toxoplasmosis among pregnant women in the Paris area. Int J Epidemiol 1988; 17:595-602.

44. Antinori A, Murri R, Tamburrini E, De Luca A, Ortona L. Failure of low-dose dapsone-pyrimethamine in primary prophylaxis of Pneumocystis carinii pneumonia. Lancet 1992;340:788.

45. Narciso P, Alba L, Antonelli L, et al. A comparison of efficacy and tolerability of four different drug regimens for primary and secondary prophylaxis of Pneumocystis carinii pneumonia (PCP) in HIV infected patients [abstract no PoB 3313]. In: Program and abstracts of the 8 th International Conference on AIDS/III STD World Congress. Amsterdam: Congrex Holland BV, 1992;2:B139.

46. Slavin MA, Hoy JF, Stewart K, Pettinger MB, Lucas CR, Kent SJ. Oral dapsone versus nebulized pentamidine for Pneumocystis carinii pneumonia prophylaxis: an open randomized prospective trial to assess efficacy and haematological toxicity. AIDS 1992;6:1169-1174.

47. Blum RN, Miller LA, Gaggini LC, Cohn DL. Comparative trial of dapsone versus trimethoprim/sulfamethoxazole for primary prophylaxis of Pneumocystis carinii pneumonia. J Acquir Immune Defic Syndr 1992; 5:341-7.

48. Salmon D, Saba J, Fontbonne A, Aboulker JP, Schwartz D, Vilde JL. Dapsone vs pentamidine aerosols for secondary prophylaxis of Pneumocystis carinii pneumonia in AIDS patients [abstract no 1474]. In: Program and abstracts of the 32nd Interscience Conference on Antimicrobial Agents and Chemotherapy. Washington, DC: American Society for Microbiology, 1992:356.

49. Jorde UP, Horowitz HW, Wormser GP. Utility of dapsone for prophylaxis of Pneumocystis carinii pneumonia in trimethoprim-sulfamethoxazole-intolerant, HIV-infected individuals. AIDS 1993; 7:355-9.

50. Bayard PJ, Berger TG, Jacobson MA. Drug hypersensitivity reactions and human immunodeficiency virus disease. J Acquir Immune Defic Syndr 1992; 5:1237-57.

51. Coopman SA, Johnson RA. Platt R, Stern RS. Cutaneous disease and drug reactions in HIV infection. N Engl J Med 1993;328:1670-4. 
52. Battegay M, Opravil M, Wüthrich B. Lüthy R. Rash with amoxycillinclavulanate therapy in HIV-infected patients [letter]. Lancet 1989;2:1100.

53. Carr A, Swanson C, Penny R, Cooper DA. Clinical and laboratory markers of hypersensitivity to trimethoprim-sulfamethoxazole in patients with Pneumocystis carinii pneumonia and AIDS. J Infect Dis 1993;167:180-5.

54. Pinching AJ, Helbert M, Peddle B. et al. Clinical experience with zidovudine for patients with acquired immune deficiency syndrome and acquired immune deficiency syndrome-related complex. J Infect 1989; 18 (suppl 1):33-40.

55. Grossman SJ, Jollow DJ. Role of dapsone hydroxylamine in dapsoneinduced hemolytic anemia. J Pharmacol Exp Ther 1988;244:11825.

56. Jacobson MA. Besch CL. Child C. et al. Primary prophylaxis with pyrimethamine for toxoplasmic encephalitis in patients with advanced human immunodeficiency virus disease: results of a randomized trial. J Infect Dis 1994; 169:384-94. 